Research Article

\title{
2.5D Inversion Algorithm of Frequency-Domain Airborne Electromagnetics with Topography
}

\author{
Jianjun $\mathrm{Xi}^{1,2}$ and Wenben $\mathrm{Li}^{1}$ \\ ${ }^{1}$ College of Geo-Exploration Science and Technology, Jilin University, Changchun 130026, China \\ ${ }^{2}$ Heibei Electric Power Design \& Research Institute, Shijiazhuang 050031, China \\ Correspondence should be addressed to Jianjun Xi; tiantianhappy365@126.com
}

Received 10 July 2016; Revised 20 August 2016; Accepted 30 August 2016

Academic Editor: Yann Favennec

Copyright (c) $2016 \mathrm{~J}$. Xi and W. Li. This is an open access article distributed under the Creative Commons Attribution License, which permits unrestricted use, distribution, and reproduction in any medium, provided the original work is properly cited.

\begin{abstract}
We presented a 2.5D inversion algorithm with topography for frequency-domain airborne electromagnetic data. The forward modeling is based on edge finite element method and uses the irregular hexahedron to adapt the topography. The electric and magnetic fields are split into primary (background) and secondary (scattered) field to eliminate the source singularity. For the multisources of frequency-domain airborne electromagnetic method, we use the large-scale sparse matrix parallel shared memory direct solver PARDISO to solve the linear system of equations efficiently. The inversion algorithm is based on Gauss-Newton method, which has the efficient convergence rate. The Jacobian matrix is calculated by "adjoint forward modelling" efficiently. The synthetic inversion examples indicated that our proposed method is correct and effective. Furthermore, ignoring the topography effect can lead to incorrect results and interpretations.
\end{abstract}

\section{Introduction}

As an important geophysical prospecting method, the frequency-domain airborne electromagnetics is widely used in fields of mineral survey, geological mapping, groundwater resource exploration, environmental monitoring, and so forth. Since the prospecting is often made in mountainous areas with large undulations which can cause severe impact upon the aeroelectromagnetic response, ignorance of the landform will lead to errors in aeroelectromagnetic data interpretation. By boundary element method, Liu and Becker simulated in 1992 the topography's impact upon the frequency-domain airborne electromagnetics [1]. Newman and Alumbaugh made 3D finite difference calculation and simulated the impact from simple topography upon the observed data results in 1995 [2]. Sasaki and Nakazato simulated the topography's impact upon the observed result by finite difference calculation and made an attempt to find a way to eliminate this impact in 2003, concluding that no way but making inversion with the topography taken into account can eliminate the impact from the topography [3]. In a word, implementing inversion with the topography taken into account is imperative in airborne electromagnetics.

The conductivity imaging technique and $1 \mathrm{D}$ layered medium inversions are still the main interpretation means in frequency-domain airborne electromagnetics [4-6]. Due to the bad reconstruction capacity of $1 \mathrm{D}$ inversion for the highdimensional models, lots of scholars made research on the inversion for high-dimensional models. Wilson and others realized 2.5D inversion in 2006 and applied it in theoretical and measured data [7]. Cox and others made 3D inversion based on footprint technique to inverse the measured data in 2010 [8]. Liu and Yin developed a finite difference 3D inversion program in 2013, compared the NLCG with LBFGS method, and drew the conclusion that the latter one is more applicable for the inversion in 3D frequency-domain airborne electromagnetic [9]. Yi and Sasaki put forward a joint inversion program making use of aeroelectromagnetic data and ground DC data in 2015 to restore the real model better and promote the inversion resolution ratio [10]. Nevertheless, all the above are conducted under the premise of a level surface, with no consideration of the topographical impact; thus in 
fact the research about inversion algorithm for the airborne electromagnetics is still quite limited.

This paper, based on vector finite element method, adopts PARDISO to solve the linear system of equations, subdivides the model in irregular hexahedral mesh, makes 3D forward modeling in topography, and employs Gauss-Newton method to carry out 2D inversion of frequency-domain airborne electromagnetics with topography, providing an effective mean to calculate, process, and interpret the aeroelectromagnetic data for mountainous areas.

\section{Forward Modeling}

To eliminate the source singularity, assume $e^{i \omega t}$ as the timeharmonic factor and divide the electromagnetic field into primary field (background) and secondary field (scattering). The double curl electric field equation based on the secondary field can be as follows [11]:

$$
\nabla \times \nabla \times \mathbf{E}_{s}+i \omega \mu_{0} \sigma \mathbf{E}_{s}=-i \omega \mu_{0} \Delta \sigma \mathbf{E}_{p}
$$

where $\mathbf{E}_{s}$ is the secondary electric field, $\omega$ is the angular frequency, $\mu_{0}$ is the magnetic conductivity in vacuum, $\sigma$ is the electric conductivity, $\mathbf{E}_{p}$ is the background field, and $\Delta \sigma$ is the difference between the total conductivity and the background conductivity, expressed as $\Delta \sigma=\sigma-\sigma_{p}$, with $\sigma_{p}$ as the background electric conductivity. The primary field under the uniform whole space medium or under the layered medium is calculated by analytic solution, while the secondary field (scattered field) is calculated by vector finite element method. The double curl Maxwell equation satisfies the following boundary condition:

$$
\begin{aligned}
\nabla \times \nabla \times \mathbf{E}_{s}+i \omega \mu_{0} \sigma \mathbf{E}_{s} & =-i \omega \mu_{0} \Delta \sigma \mathbf{E}_{p}, \quad \mathbf{E}_{s} \in \Omega \\
\mathbf{E}_{s} & =0, \quad \mathbf{E}_{s} \in \partial \Gamma .
\end{aligned}
$$

Implement the Galerkin weighted residual method for formula (1); then

$$
\begin{aligned}
R_{k} & =\int_{\Omega} \mathbf{N}_{k} \cdot\left[\nabla \times \nabla \times \mathbf{E}_{s}+i \omega \mu \sigma \mathbf{E}_{s}+i \omega \mu \Delta \sigma \mathbf{E}_{p}\right] d V \\
& =0 .
\end{aligned}
$$

In the equation $\Omega=\sum_{e=1}^{N_{e}} \Omega^{e}, \Omega^{e}$ represents a discrete element and $N_{e}$ is the number of discrete elements; thus formula (3) can be expressed in discrete form as below:

$$
R_{k}=\sum_{e=1}^{N_{e}}\left[\mathbf{K}^{e} \mathbf{E}_{s}^{e}-i \omega \mu \sigma \mathbf{M}^{e} \mathbf{E}_{s}^{e}-i \omega \mu \Delta \sigma \mathbf{M}^{e} \mathbf{E}_{p}^{e}\right]=0
$$

where $\mathbf{K}^{e}$ and $\mathbf{M}^{e}$ are element stiffness matrix, expressed as

$$
\begin{aligned}
\mathbf{K}_{k l}^{e} & =\int_{\Omega^{e}}\left(\nabla \times \mathbf{N}_{k}^{e}\right) \cdot\left(\nabla \times \mathbf{N}_{l}^{e}\right) d V \\
\mathbf{M}_{k l}^{e} & =\int_{\Omega^{e}} \mathbf{N}_{k}^{e} \cdot \mathbf{N}_{l}^{e} d V .
\end{aligned}
$$
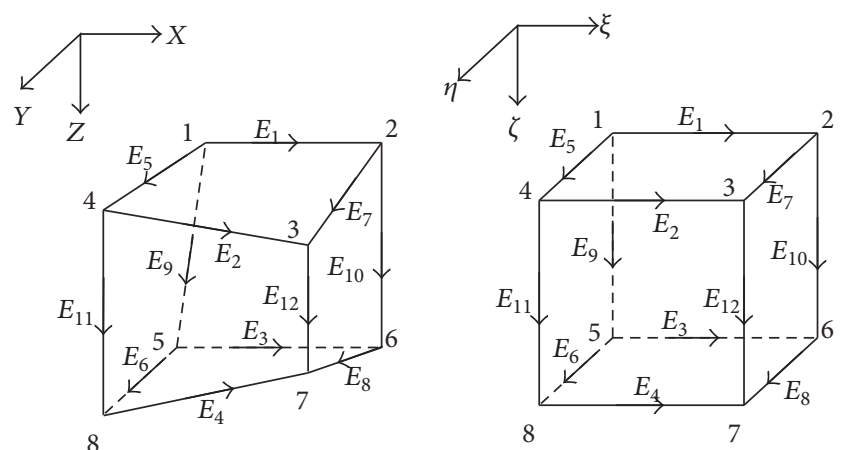

FIGURE 1: Element global coordinates mapping on local coordinates.

$\mathbf{N}_{k}^{e}$ is the vector basis function [12]. 27-point Gauss integral is used for the calculations in formula (5).

The element global coordinates mapping on local coordinates is shown in Figure 1. Allocate the element stiffness matrix to the overall stiffness matrix according to certain standard, and then the large-scale linear system of equations can be drawn:

$$
\mathbf{K F}=\mathbf{b} .
$$

$\mathbf{K}$ is sparse complex symmetric matrix, $\mathbf{F}$ is the field value, and $\mathbf{b}$ is the source item.

To guarantee a unique solution, the Dirichlet boundary condition is employed, deeming that the secondary abnormal field at the boundary which is far enough from the anomalous body has attenuated nearly to zero. In other words, at the boundary,

$$
\mathbf{n} \times\left.\mathbf{E}\right|_{\partial \Omega}=\mathbf{0} .
$$

Once the electric field is calculated, the magnetic field can be solved by Faraday's law:

$$
\mathbf{H}=\left(-i \omega \mu_{0}\right)^{-1} \nabla \times \mathbf{E}
$$

2.1. Precision Validation of Forward Modeling. To verify the correctness of this procedure, a 2D trapezoid mountain model the same as that used by Sasaki and Nakazato [3] is adopted with the medium electric conductivity of $100 \mathrm{ohm}$ $\mathrm{m}$, as shown in Figure 2. The top and bottom width of the trapezoid are, respectively, $20 \mathrm{~m}$ and $220 \mathrm{~m}$, the height of the trapezoid is $50 \mathrm{~m}$, and the gradient is $26.5^{\circ}$. Subdivide the modeling area in $x, y$, and $z$ direction into $41 \times 41 \times 41$ grids, with the one in the middle sized $10 \mathrm{~m} \times 10 \mathrm{~m} \times 10 \mathrm{~m}$ and the largest one at the boundary sized $1280 \mathrm{~m} \times 1280 \mathrm{~m} \times 1280 \mathrm{~m}$. HCP device is employed for calculation, with the transmitterreceiver coil pair keeping $30 \mathrm{~m}$ above the ground in $10 \mathrm{~m}$ transmitter-receiver spacing in three frequencies of $1 \mathrm{kHz}$, $4 \mathrm{kHz}$, and $16 \mathrm{kHz}$. The results in Figure 3 highly coincide with Sasaki's finite difference method results, which illustrated that the forward modeling program in this paper is highly accurate. 


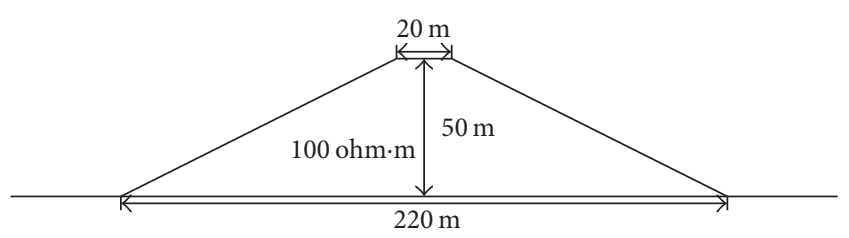

FIGURE 2: 2D trapezoid mountain model diagram.

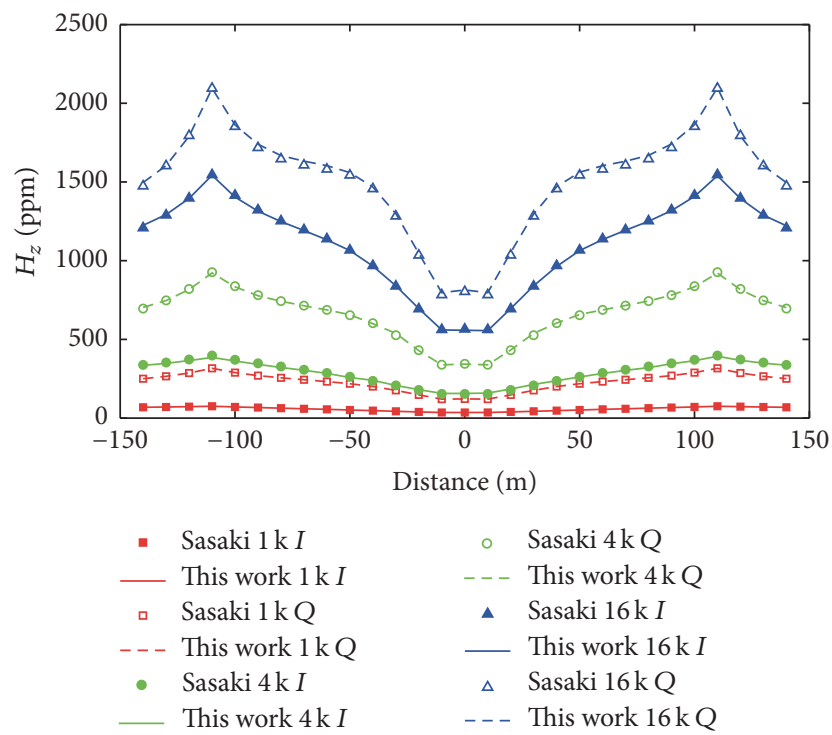

FIGURE 3: Comparison with Sasaki’s finite difference method result.

\section{Inversion Algorithm}

3.1. Objective Function Definition. The objective function can be written as

$$
\Phi(\mathbf{m})=\frac{1}{2}\left\|\mathbf{d}(\mathbf{m})-\mathbf{d}_{\text {obs }}\right\|^{2}+\frac{\beta}{2}\left\|\mathbf{W}\left(\mathbf{m}-\mathbf{m}_{\mathrm{ref}}\right)\right\|^{2},
$$

where $\|\cdot\|$ denotes the Euclidean norm, $\mathbf{d}$ is the data vector obtained from forward modeling, $\mathbf{d}_{\mathrm{obs}}$ is the measured data vector, $\mathbf{m}$ is the model parameter vector, $\mathbf{m}_{\text {ref }}$ is the reference model vector or the prior information model vector, $\mathbf{W}$ is the model smoothness matrix, and $\beta$ is the regularization parameter that balances the effect of data misfit and model regularization during minimization.

The objective function defined by formula (9) is divided into data fitting item and regularization item. The former guarantees the inversed model to fit the measured data, while the latter controls the inversion result to draw close to the known prior model. There are normally three cases for the regularization item: (1) smallest model, that is, smallest $\mathbf{m}$ $\mathbf{m}_{\text {ref }}$; (2) flattest model, that is, smallest first-order difference operator for $\mathbf{m}-\mathbf{m}_{\text {ref }}$; (3) most smooth model, that is, smallest second-order difference operator for $\mathbf{m}-\mathbf{m}_{\text {ref. }}$. Due to the inversion algorithm in this paper, the third type is adopted, that is, with $\mathbf{W}$ as the second-order difference operator, and has the following form:

$$
\mathbf{W}=\left[\begin{array}{cccccccccc}
-4 & 1 & 0 & \cdots & 1 & 0 & \cdots & 0 & \cdots & 0 \\
1 & -4 & 1 & 0 & \cdots & 1 & 0 & \cdots & 0 & \cdots \\
\vdots & & & & & & & & & \vdots \\
0 & \cdots & 0 & 1 & 0 & \cdots & 0 & 1 & -4 & 1 \\
0 & \cdots & 0 & \cdots & 0 & 1 & \cdots & 0 & 1 & -4
\end{array}\right] .
$$

Assuming $\mathbf{m}_{i}$ as the initial model, substitute it into formula (9) and linearize it by Taylor expansion, ignoring the higher order terms to reach

$$
\begin{aligned}
\Phi(\mathbf{m})= & \frac{1}{2}\left\|\left(\mathbf{d}+\frac{\partial \mathbf{d}}{\partial \mathbf{m}_{i}} \cdot \Delta \mathbf{m}\right)-\mathbf{d}_{\mathrm{obs}}\right\|^{2} \\
& +\frac{\beta}{2}\left\|\mathbf{W}\left(\mathbf{m}_{i}-\mathbf{m}_{\text {ref }}\right)\right\|^{2}
\end{aligned}
$$

where $\Delta \mathbf{m}$ is $\mathbf{m}-\mathbf{m}_{i}$. Derive formula (11) and order it equal to zero so as to obtain the model updated iterative formula for Gauss-Newton method:

$$
\begin{aligned}
& \underbrace{\left(\mathbf{J}^{T} \mathbf{J}+\beta \mathbf{W}^{T} \mathbf{W}\right)}_{H} \cdot \Delta \mathbf{m} \\
& =\underbrace{-\left(\mathbf{J}^{T}\left(\mathbf{d}-\mathbf{d}_{\mathrm{obs}}\right)+\beta \mathbf{W}^{T} \mathbf{W}\left(\mathbf{m}-\mathbf{m}_{\mathrm{ref}}\right)\right)}_{g},
\end{aligned}
$$

where $H$ is the approximate Hessian matrix, $g$ is the objective function gradient, and $\mathbf{J}$ is Jacobian matrix or sensitivity matrix, with its elements expressed as

$$
\mathbf{J}=\left[\begin{array}{ccc}
\frac{\partial d_{1}}{\partial m_{1}} & \cdots & \frac{\partial d_{1}}{\partial m_{n}} \\
\vdots & \ddots & \vdots \\
\frac{\partial d_{m}}{\partial m_{1}} & \cdots & \frac{\partial d_{m}}{\partial m_{n}}
\end{array}\right]
$$

3.2. Jacobian Matrix Calculation. From formula (12), it is known that the key to the model update iteration is to solve the linear system of equations, which in turn is decided by Jacobian matrix or sensitivity matrix $\mathbf{J}$; thus the calculation for Jacobian matrix is crucial in the inversion algorithm, and its calculation efficiency is decisive to some extent for the inversion efficiency. The calculation methods for Jacobian matrix include analytic method, difference method, and adjoint equation solution method [13-15]. Since the analytic method cannot solve the matrix in high-dimensional inversion and the difference method, though simple, is inefficient, here we choose to solve the adjoint equation for the solution of Jacobian matrix. The calculation formula for the magnetic field is expressed as

$$
\mathbf{H}=\left(-i \omega \mu_{0}\right)^{-1} \sum_{i=1}^{12} \nabla \times \mathbf{N}_{i} E_{i} .
$$


The magnetic field's model parameter derivative is expressed as

$$
\frac{\partial \mathbf{H}}{\partial m}=\left(-i \omega \mu_{0}\right)^{-1} \sum_{i=1}^{12} \nabla \times \mathbf{N}_{i} \frac{\partial E_{i}}{\partial m} .
$$

The magnetic field's model parameter derivative can be converted into the electric field's model parameter derivative. The forward modeling can yield a large-scale complex number linear system of equations:

$$
\mathbf{A x}=\mathbf{b}
$$

Derive $m$ simultaneously from both sides of formula (15) to work out

$$
\mathbf{A} \frac{\partial \mathbf{x}}{\partial m}=-\frac{\partial \mathbf{A}}{\partial m} \mathbf{x}+\frac{\partial \mathbf{b}}{\partial m}
$$

The electric field's model parameter derivative can be worked out by solving formula (17). Formula (17) has nearly the same coefficient matrix $\mathbf{A}$ as the forward modeling linear system of equations, with difference at the right-hand item. The solving process is similar to the forward modeling process, named as "accompanying forward modeling." According to interchange theorem, to obtain the model parameter sensitivity of the magnetic field at the receiving point, all that is needed is to put the unit source at the receiving point and conduct "accompanying forward modeling" once. It is equivalent to the simple weighted summation with the model parameter-correlated element field value when putting the unit source at the exact receiving point, which can be worked out from the right-hand item of formula (17). "Accompanying forward modeling" is needed to be carried out just once, which refers to solving all the measured points and frequency points, and then the Jacobian matrix can be obtained.

3.3. Solution for Model Updated Equations. As known from formula (12), to obtain the model parameter update vector quantity, the linear system of equations must be solved first, either solved directly or solved by iterative method. $\mathbf{J}^{T} \mathbf{J}$ needs be worked out for the solution of formula (12). If selecting direct solution, Jacobian matrix $\mathbf{J}$ is needed to be stored. Here the preconditioned conjugate gradient iteration method is adopted, only with the multiplication of the matrix and the vector required to be worked out. As for whether to store the Jacobian matrix in the iteration method, if storing, though unnecessary to solve $\mathbf{J}$ in each time of iteration, the memory demand will be large, and Jacobian matrix must be stored entirely due to its denseness, while if not storing, the memory insufficiency can be avoided, but for each time of iteration, Jacobian matrix must be calculated once again, plus the forward modeling and "accompanying forward modeling" being conducted at each time.

3.3.1. Step Length Selection. After obtaining the model parameter update vector $\Delta \mathbf{m}$ by working out formula (12), the next iterative model is updated by

$$
\mathbf{m}_{i+1}=\mathbf{m}_{i}+\alpha \Delta \mathbf{m},
$$

where $\alpha$ is the step length with the range as $0<\alpha \leq 1$. The suitable step length is defined by the following steepest descent formula [16]:

$$
\begin{aligned}
\phi\left(\mathbf{m}_{i+1}\right) & =\phi\left(\mathbf{m}_{i}+\alpha \Delta \mathbf{m}\right) \\
& \leq \phi\left(\mathbf{m}_{i}\right)+c_{1} \alpha \nabla \phi\left(\mathbf{m}_{i}\right) \Delta \mathbf{m},
\end{aligned}
$$

where $c_{1}$ is a constant that in practice takes a very small value, for example, $10^{-4}$. Satisfying (19) guarantees that the our new model update adequately reduces the objective function. When performing the model update in (18), the initial value of $\alpha$ is set to one and the new model is generated. The inequality in (19) was evaluated, and if the condition is satisfied, the new model is acceptable., However, if the condition is not satisfied, $\alpha$ is reduced by one-half, and (19) was reevaluated. This procedure is repeated until we find a new model that meets the criterion of (19).

The iterative process is repeated until the objective function and/or the data misfit have reduced to a specified level. Alternatively, the inversion can be stopped when the magnitude of the objective functional gradient falls below a given tolerance, for example, $1.0 e-10$, which indicates that the objective function has flattened out and that no further model improvements can be made. Moreover, the inversion can be stopped when the iteration number exceeds the maximum iteration number. Our experience suggests that it is usually not more than ten iterations. Otherwise, stopping the inversion once the data misfit reaches the noise level of the measure data usually provides reasonable results.

3.3.2. Regularization Factor Selection. The regularization factor $\beta$ can balance the data misfit and model constraints. There are lots of schemes that have been recommended in literature and used in practice for the selection of $\beta[17,18]$. To deal with $\beta$, there are three most common approaches: (1) one fixed $\beta$ value in the whole inversion process; (2) decreasing $\beta$ value gradually in each time of iteration; (3) choosing the most appropriate $\beta$ value by $L$ curve in each time of iteration. Normally, scheme (3) has the best effect but requires additional calculation work to seek the appropriate value each time. In this paper, schemes (1) and (2) are adopted in the inversion and are found capable of yielding satisfactory results as well. Furthermore, for a given problem one must often determine $\beta$ through trial and error. When using trial and error, one should seek the largest $\beta$ value which still permits that data to be fit down to the noise level.

3.4. Inversion Process. The inversion procedure is shown in Figure 4:

(1) Set the number of iterations as $i=0$, the accuracy of fitting, and the largest number of iterations, and input the initial model and inversion data.

(2) Conduct the forward modeling and solve the forward modeling equation $\mathbf{K F}=\mathbf{b}$ to work out the secondary magnetic field $H_{x}$ and $H_{z}$.

(3) Calculate the fitting error, exit the program if the set accuracy or the largest number of iterations is achieved, and continue the program if not. 


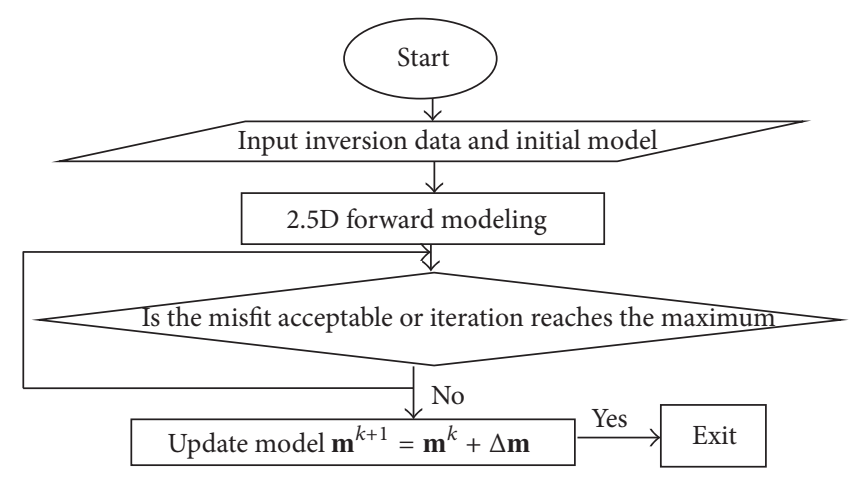

FIGURE 4: Inversion process.

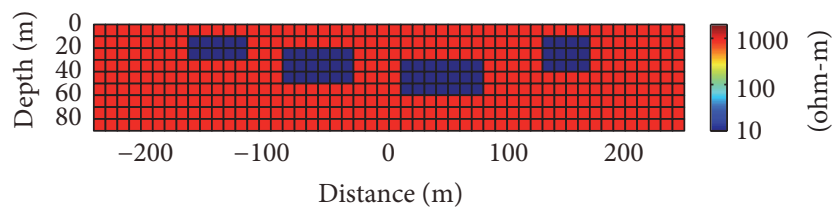

FIgURE 5: Multiple anomalous bodies inversion model.

(4) Calculate Jacobian matrix by forward simulation to obtain the updated step length.

(5) Update the model parameter: $\mathbf{m}^{k+1}=\mathbf{m}^{k}+\Delta \mathbf{m}$.

\section{Synthetic Inversion Examples}

The synthetic data sets from two $2 \mathrm{D}$ models were used to test the inversion algorithm. The first is a flat-earth model. The second example represents a 2D feature with topography. The data sets are generated for frequency-domain airborne electromagnetic with HCP or VCX using the vector finite element method.

4.1. Inversion in Flat-Earth Model. Figure 5 shows the model used to test the inversion method for flat-earth model. There are four anomalies bodies with $10 \mathrm{ohm}-\mathrm{m}$ resistivity buried in $1000 \mathrm{ohm}-\mathrm{m}$ half-space. HCP and VCX devices are employed under the frequencies of $1000 \mathrm{~Hz}, 2700 \mathrm{~Hz}, 7400 \mathrm{~Hz}$, and $20000 \mathrm{~Hz}$. The coil pair is keeping $20 \mathrm{~m}$ above the ground with the transmitter-receiver distance of $8 \mathrm{~m}$, and there are totally 51 dots with spacing of $10 \mathrm{~m}$. The model domain is subdivided into $69 \times 34 \times 29$ grids, with the one in the middle sized $10 \mathrm{~m} \times 10 \mathrm{~m} \times 10 \mathrm{~m}$ and the largest one at the boundary sized $1280 \mathrm{~m} \times 1280 \mathrm{~m} \times 1280 \mathrm{~m}$. The forward modeling results for HCP and VCX are shown in Figures 6 and 7, respectively, with the value normalized by the primary field in ppm.

The inversion model is subdivided into $52 \times 1 \times 10$ pieces, with the 520 electric resistance unknown, while the initial model is even half-spatial with resistance of $300 \mathrm{ohm}-\mathrm{m}$. By 10 times of iterations, HCP and VCX are converged to $1.5 \%$ and $0.7 \%$ of the origin, respectively. Figure 8 shows the RMS variation curve along with the increasing iterations for
HCP and VCX device. Their inversion results are shown in Figures 9 and 10, respectively, from which it is observed that both HCP and VCX have satisfactorily reconstructed the real model. Comparatively, HCP has better lateral resolution while VCX acts better in vertical resolution.

4.2. Inversion in the Model with Topography. The following two models are designed to analyze and check the inversion effect in undulating surface condition.

4.2.1. Low Resistance Composite Pattern. The model is an even half-spatial mountain type as shown in Figure 11, with two low resistance anomalous bodies buried therein. The electric resistance of the anomalous bodies and of the background is, respectively, $10 \mathrm{ohm}-\mathrm{m}$ and $300 \mathrm{ohm}-\mathrm{m}$. The model top is $20 \mathrm{~m}$ wide and $50 \mathrm{~m}$ from the bottom, and the gradient is $26.6^{\circ}$. Here we change the coordinate of $z$-axis in the grids to simulate the undulating surface. HCP device is employed under the frequencies of $1000 \mathrm{~Hz}, 2700 \mathrm{~Hz}$, $7400 \mathrm{~Hz}$, and $20000 \mathrm{~Hz}$. The coil is $20 \mathrm{~m}$ above the ground with transmit-receive distance of $8 \mathrm{~m}$, and there are totally 51 dots in spacing of $10 \mathrm{~m}$. The model domain is subdivided into $69 \times 34 \times 29$ grids, with the one in the middle sized $10 \mathrm{~m} \times 10 \mathrm{~m} \times 10 \mathrm{~m}$ and the largest one at the boundary sized $1280 \mathrm{~m} \times 1280 \mathrm{~m} \times 1280 \mathrm{~m}$. The forward modeling results for HCP are shown in Figure 12, with the value normalized by the primary field in ppm.

In the inversion, the forward modeling uses a grid of $69 \times 34 \times 29$ cells. The inversion model region is subdivided into $52 \times 1 \times 10$ pieces, with the 520 unknown electric resistivity, and the starting model is half-space with resistance of $300 \mathrm{ohm}-\mathrm{m}$. After 10 iterations, the HCP RMS is reduced to $0.5 \%$ of the first iteration. The RMS variation with the increasing of iteration is shown in Figure 13. The inversion result is shown in Figure 14, from which it is observed that HCP has satisfactorily reconstructed the real model.

To illustrate the effectiveness of the inversion algorithm with the landform being taken into account, the inversion results for different landforms are shown in Figure 15. It is observed that ignorance of the landform will cause undesirable inversion result; for example, the low resistance anomalous body, though being reflected, is far different from the real 


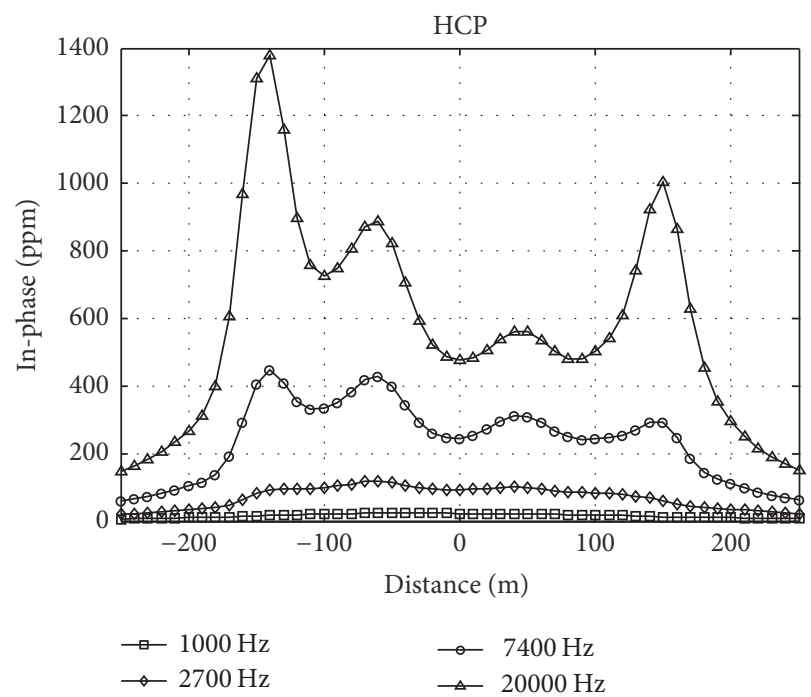

(a) Real component

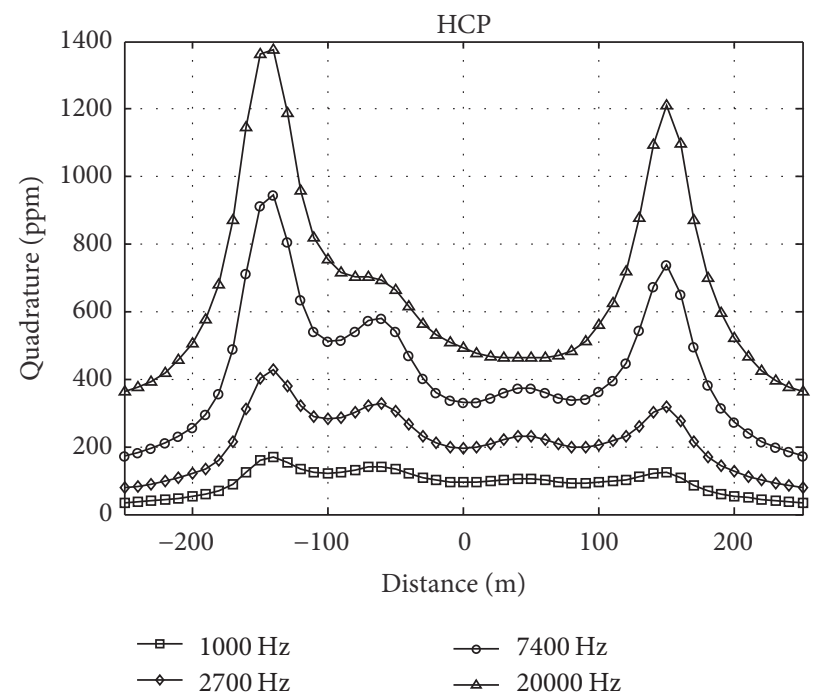

(b) Imaginary component

FIGURE 6: HCP forward modeling result.

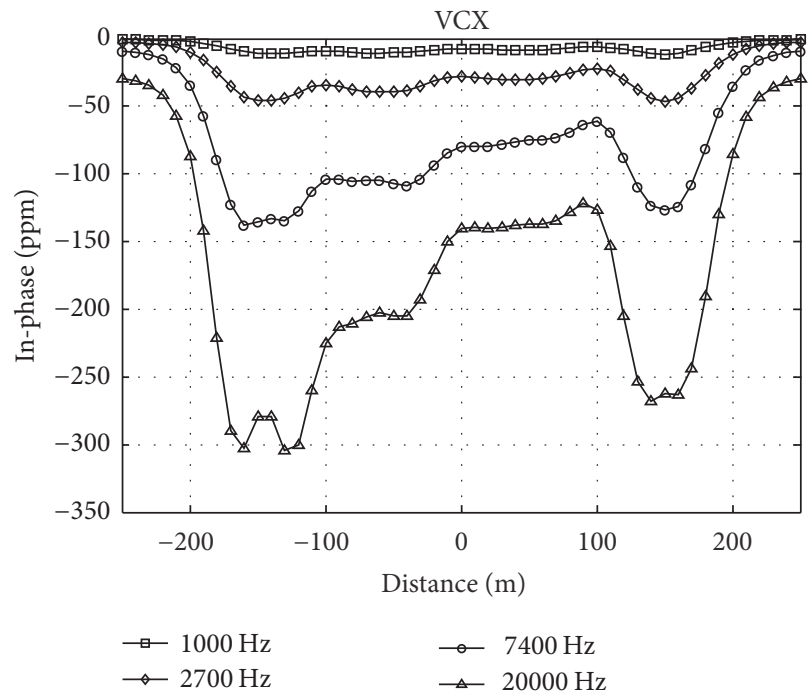

(a) Real component

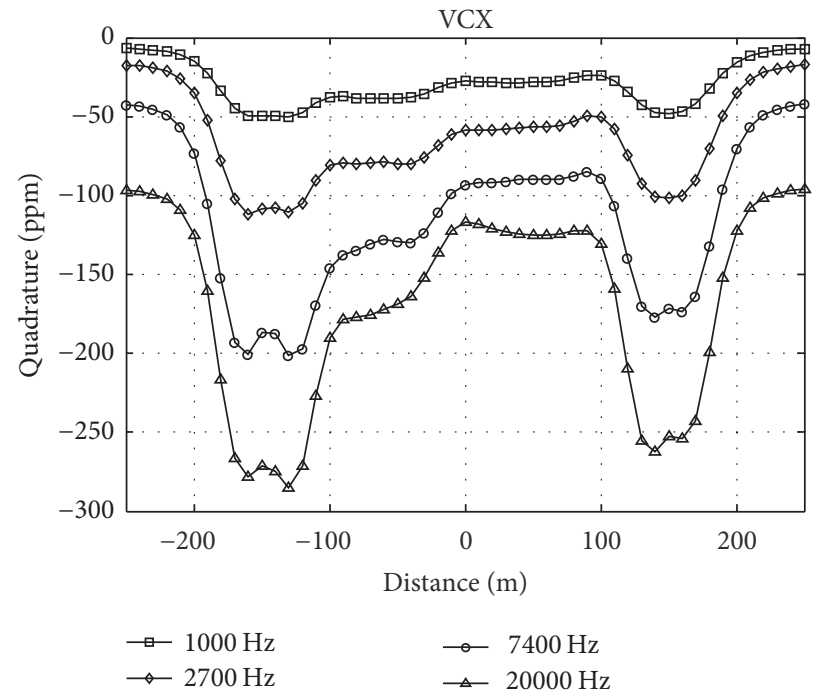

(b) Imaginary component

FIGURE 7: VCX forward modeling result.

model, especially for the lateral stretch. Besides, much falseness and abnormities arise in the inversion profile, and particularly a high resistance anomalous body arises right below the mountain due to the topographic impact.

4.2.2. High-Low Resistance Composite Pattern. The high-low resistance composite pattern is shown in Figure 16, with the anomalous bodies in $10 \mathrm{ohm}-\mathrm{m}$ low and $3000 \mathrm{ohm}-\mathrm{m}$ high resistance. The other parameters are the same as those in low resistance composite pattern. The forward modeling result is shown in Figure 17.

The initial model for inversion is even half-spatial with resistance of $300 \mathrm{ohm}-\mathrm{m}$. By 10 times of iterations, the fitting difference reduces to $2.2 \%$ of the first iteration misfit, referring to Figure 18 for its variation along with the iterations. From Figure 19 showing inversion result for high-low resistance composite pattern (with the landform being taken into account), it is observed that the inversion result has satisfactorily reconstructed the real geoelectric model with no redundant abnormity, whereas the high resistance anomalous body, though being located correctly, is observed with its resistance value as just $600 \mathrm{ohm}-\mathrm{m}$ which is far different from the real value of $3000 \mathrm{ohm}-\mathrm{m}$. Oldenburg (2012) and others have asserted that this phenomenon is normal [19], because the pure secondary abnormal field collected by frequency-domain airborne electromagnetics is insensitive 


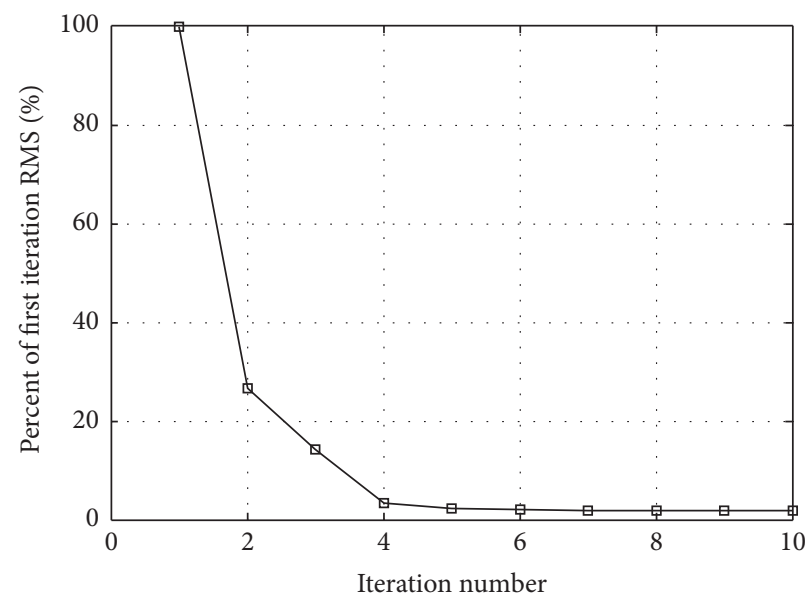

(a) HCP device

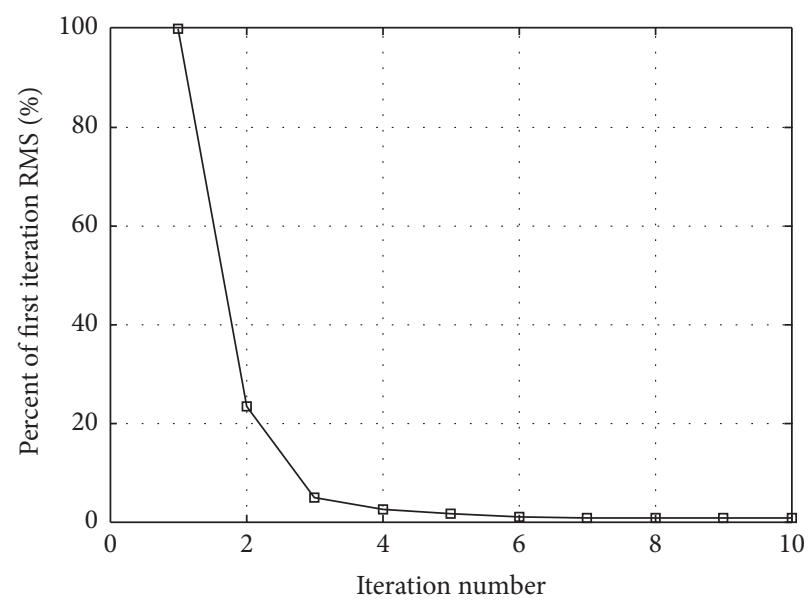

(b) VCX device

FIGURE 8: RMS variation along with the times of iterations.

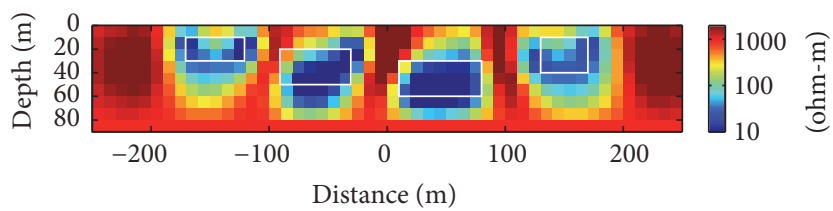

FIGURE 9: HCP inversion result.

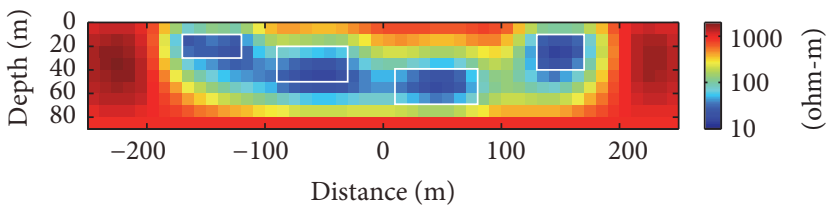

FIGURE 10: VCX inversion result.

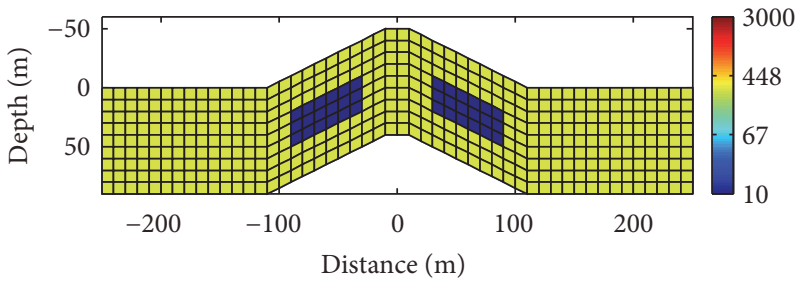

FIGURE 11: Mountain low resistance composite pattern.

to high resistance body, and, consequently, the inductive source magnetic field data is essentially difficult to inverse the high resistance body. In Figure 20 showing inversion result for high-low resistance composite pattern (without taking into account the landform), it is observed that the inversion result, though capable of reflecting the outline of high or low resistance anomalous bodies, is far different from the real model, particularly with large lateral stretch arising for the low resistance anomalous body. Moreover, the same as the low resistance composite pattern, much falseness and abnormities arise, and particularly a high resistance anomalous body arises right below the mountain.

\section{Conclusion}

We have developed the $2.5 \mathrm{D}$ inversion algorithm for frequency-domain airborne electromagnetic data and applied it to synthetic data, such as flat-earth model and the model with topography. Commencing from analysis for the inversion effect of HCP and VCX device at horizontal landform, the paper makes research for multiple low resistance composite pattern of anomalous bodies and indicates that HCP has better lateral resolution while VCX acts better in vertical resolution. Therefore, data for various measuring devices shall be made use of to promote the inversion efficiency. Afterwards, through research for the mountain low resistance composite pattern and high-low resistance composite pattern, it is concluded that inversion with the topography being 


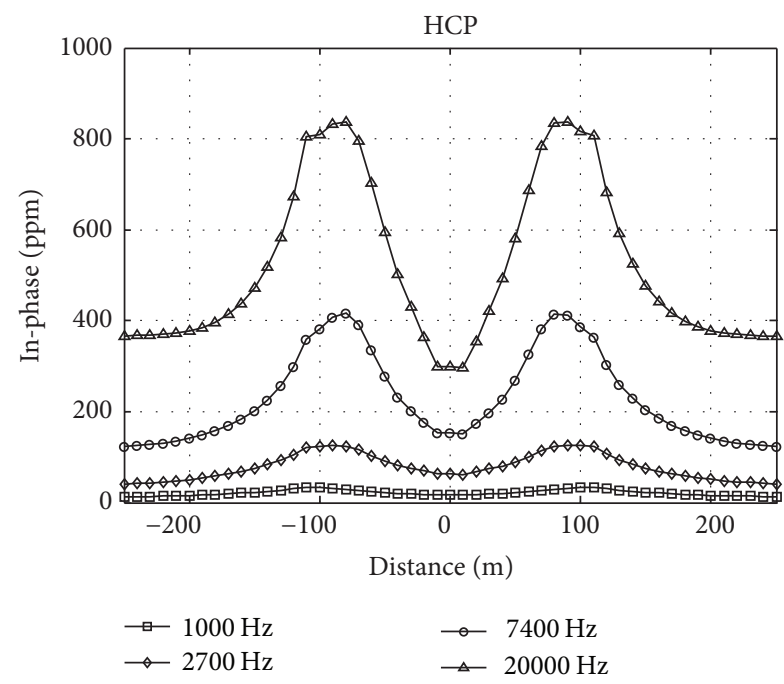

(a) Real component

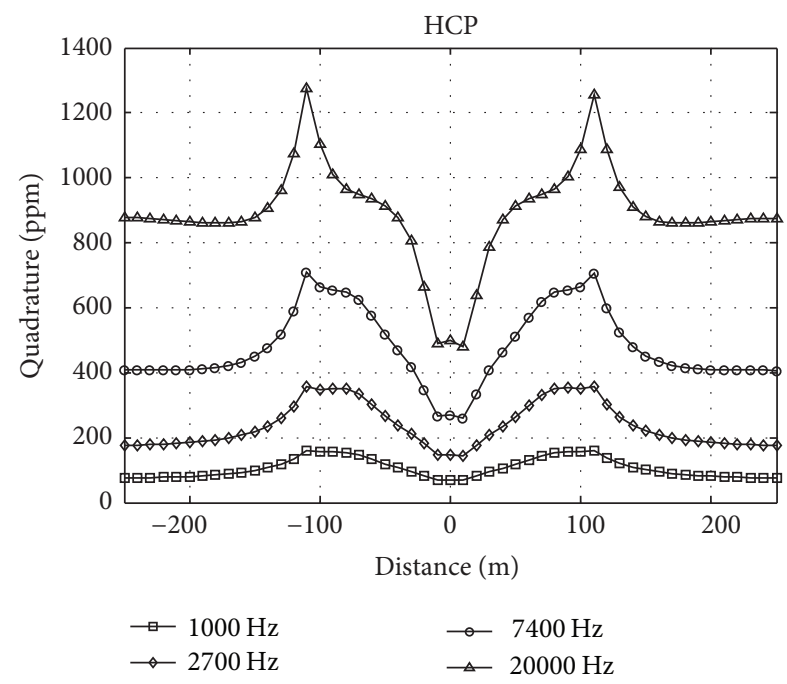

(b) Imaginary component

FIGURE 12: HCP forward modeling result.

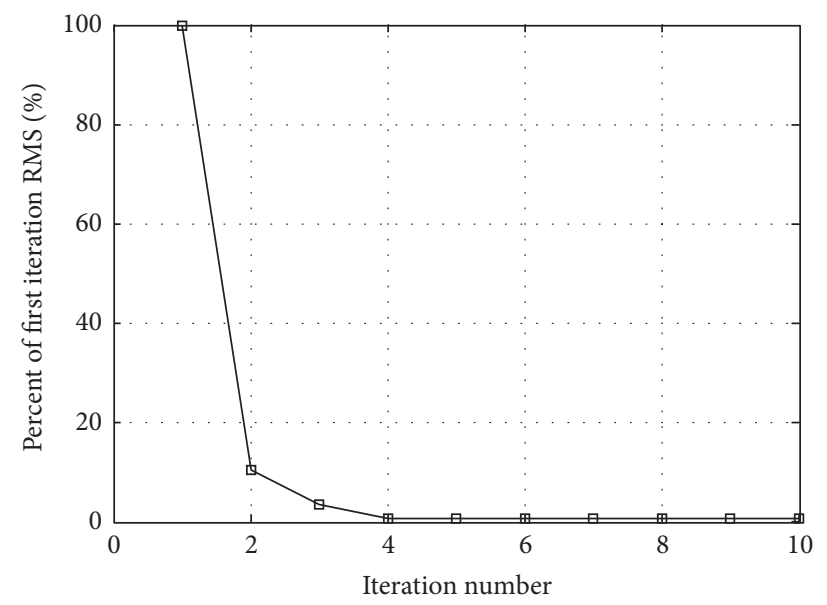

FIGURE 13: RMS variation along with the times of iterations.

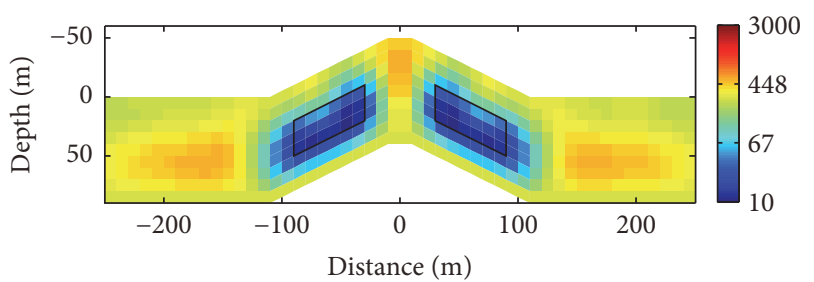

FIGURE 14: Inversion result for mountain low resistance composite pattern (with the landform being taken into account).

taken into account can better reconstruct the real model; otherwise much falseness and abnormities will arise, and particularly a high resistance anomalous body will arise right below the mountain. Thus to obtain an accurate geoelectric model, the impact from the topography must be taken into account in the measured data processing.

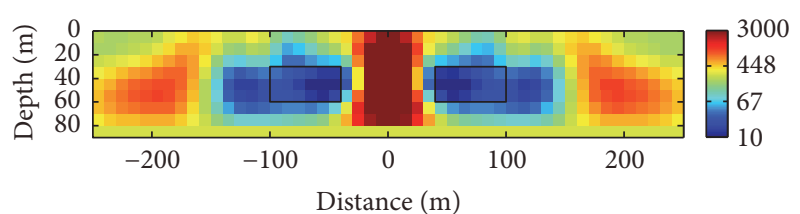

FIGURE 15: Inversion result for mountain low resistance composite pattern (without taken into account the landform).

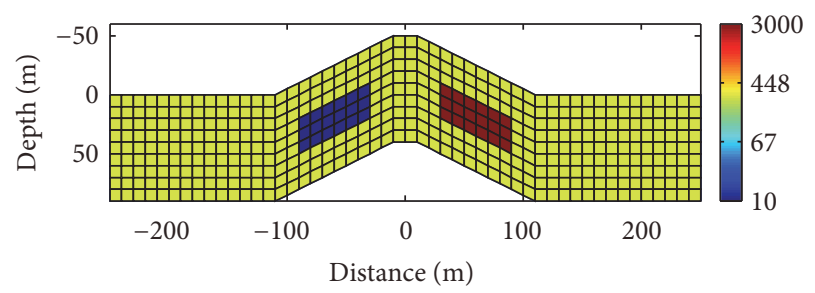

FIGURE 16: Mountain high-low resistance composite pattern. 


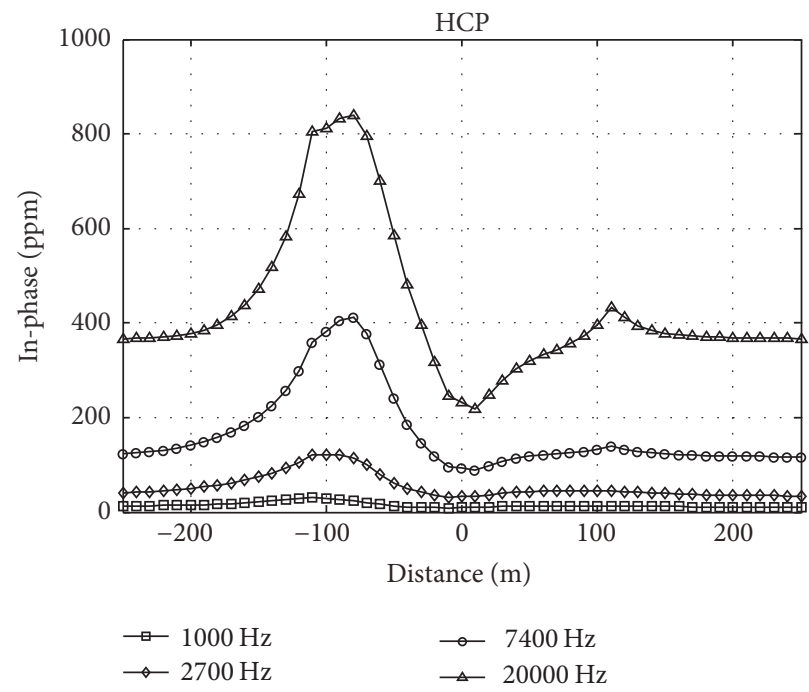

(a) Real component

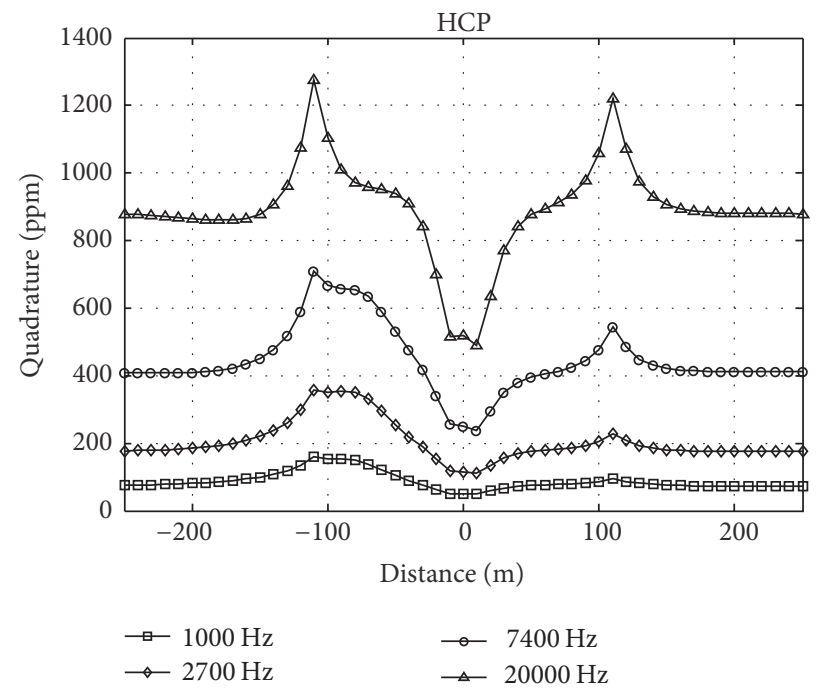

(b) Imaginary component

FIGURE 17: HCP forward modeling result.

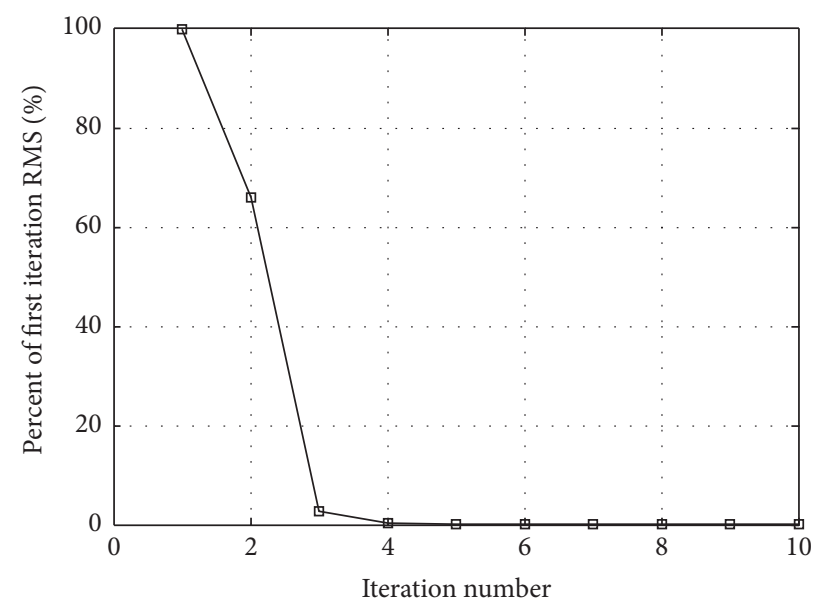

FIGURE 18: RMS variation along with the times of iterations.

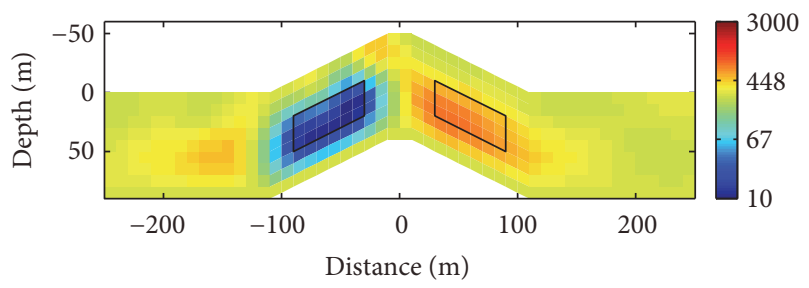

FIGURE 19: Inversion result for high-low resistance composite pattern (with the landform being taken into account).

\section{Competing Interests}

The authors declare that they have no competing interests.

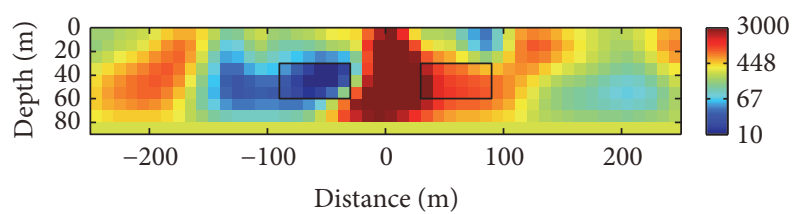

FIGURE 20: Inversion result for high-low resistance composite pattern (without taking into account the landform).

\section{Acknowledgments}

The authors thank the financial support of the Doctoral Fund Project of the Ministry of Education (no. 20130061110060, class tutors), the National Natural Science Foundation of China (no. 41504083), and National Basic Research Program of China (973 Program) (no. 2013CB429805).

\section{References}

[1] G. M. Liu and A. Becker, "Evaluation of terrain effects in AEM surveys using the boundary element method," Geophysics, vol. 57, no. 2, pp. 272-278, 1992.

[2] G. A. Newman and D. L. Alumbaugh, "Frequency-domain modelling of airborne electromagnetic responses using staggered finite differences," Geophysical Prospecting, vol. 43, no. 8, pp. 1021-1042, 1995.

[3] Y. Sasaki and H. Nakazato, "Topographic effects in frequencydomain helicopter-borne electromagnetics," Exploration Geophysics, vol. 34, no. 2, pp. 24-28, 2003.

[4] C. G. Farquharson, D. W. Oldenburg, and P. S. Routh, "Simultaneous 1D inversion of loop-loop electromagnetic data for magnetic susceptibility and electrical conductivity," Geophysics, vol. 68, no. 6, pp. 1857-1869, 2003. 
[5] H. Huang and D. C. Fraser, "The differential parameter method for multifrequency airborne resistivity mapping," Geophysics, vol. 61, no. 1, pp. 100-109, 1996.

[6] C. C. Yin and G. Hodges, "Simulated annealing for airborne EM inversion," Geophysics, vol. 72, no. 4, pp. F189-F195, 2007.

[7] G. A. Wilson, A. P. Raiche, and F. Sugeng, "2.5D inversion of airborne electromagnetic data," Exploration Geophysics, vol. 37, no. 4, pp. 363-371, 2006.

[8] L. H. Cox, G. A. Wilson, and M. S. Zhdanov, "3D inversion of airborne electromagnetic data using a moving footprint," Exploration Geophysics, vol. 41, no. 4, pp. 250-259, 2010.

[9] Y.-H. Liu and C.-C. Yin, "3D inversion for frequency-domain HEM data," Chinese Journal of Geophysics, vol. 56, no. 12, pp. 4278-4287, 2013.

[10] M. Yi and Y. Sasaki, "2-D and 3-D joint inversion of loop-loop electromagnetic and electrical data for resistivity and magnetic susceptibility," Geophysical Journal International, vol. 203, no. 2, pp. 1085-1095, 2015.

[11] R. Streich, "3D finite-difference frequency-domain modeling of controlled-source electromagnetic data. Direct solution and optimization for high accuracy," Geophysics, vol. 74, no. 5, pp. F95-F105, 2009.

[12] J. Jin, The Finite Element Method in Electromagnetics, WileyIEEE Press, New York, NY, USA, 2002.

[13] P. R. McGillivray, D. W. Oldenburg, R. G. Ellis, and T. M. Habashy, "Calculation of sensitivities for the frequency-domain electromagnetic problem," Geophysical Journal International, vol. 116, no. 1, pp. 1-4, 1994.

[14] P. Weidelt, "Inversion of two-dimensional conductivity structures," Physics of the Earth and Planetary Interiors, vol. 10, no. 3, pp. 282-291, 1975.

[15] P. P. De Lugão and P. E. Wannamaker, "Calculating the twodimensional magnetotelluric Jacobian in finite elements using reciprocity," Geophysical Journal International, vol. 127, no. 3, pp. 806-810, 1996.

[16] L. Armijo, "Minimization of functions having Lipschitz continuous first partial derivatives," Pacific Journal of Mathematics, vol. 16, pp. 1-3, 1966.

[17] S. C. Constable, R. L. Parker, and C. G. Constable, "Occam's inversion: a practical algorithm for generating smooth models from electromagnetic sounding data," Geophysics, vol. 52, no. 3, pp. 289-300, 1987.

[18] P. C. Hansen, "Rank-deficient and discrete ill-posed problems: numerical aspects of linear inversion," Society of Industrial and Applied Mathematics, vol. 4, no. 5, pp. 491-491, 1997.

[19] D. W. Oldenburg and Y. Li, "Estimating depth of investigation in dc resistivity and IP surveys," Geophysics, vol. 64, no. 64, pp. 403-416, 2012. 


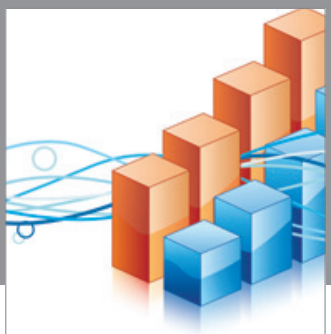

Advances in

Operations Research

vatem alat4

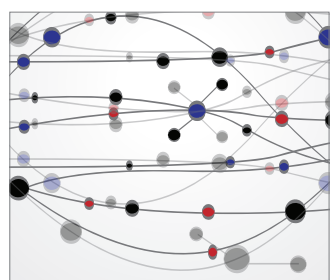

\section{The Scientific} World Journal
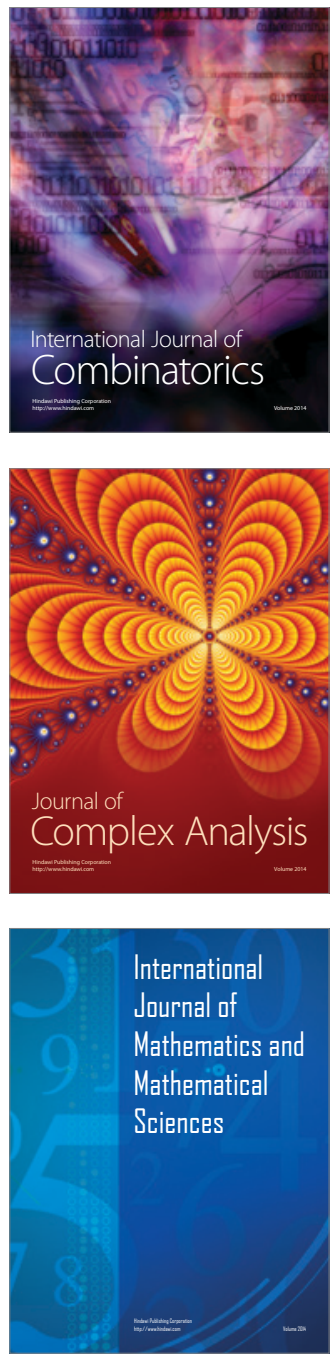
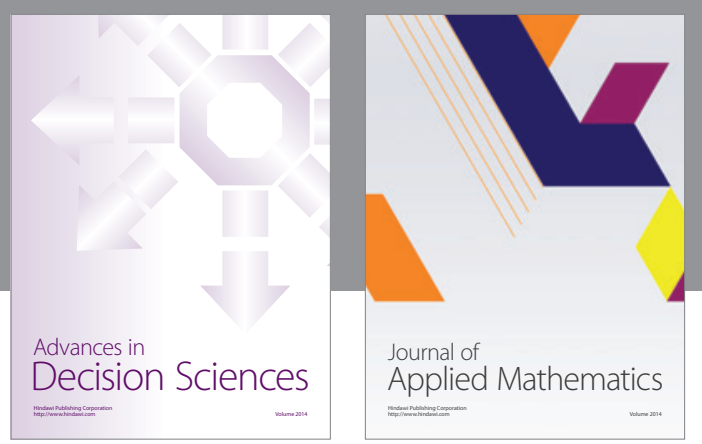

Algebra

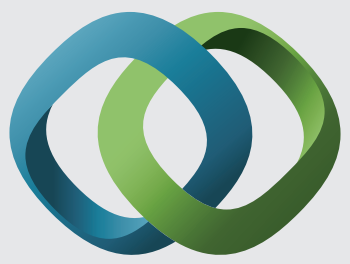

\section{Hindawi}

Submit your manuscripts at

http://www.hindawi.com
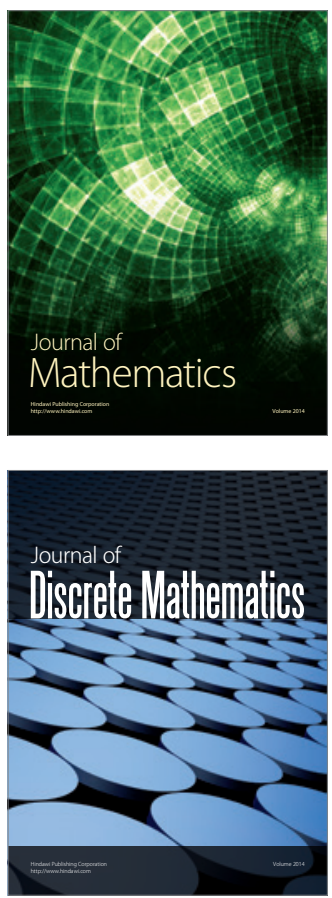

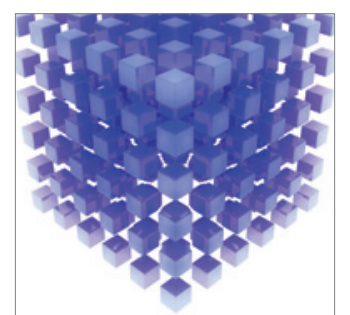

Mathematical Problems in Engineering
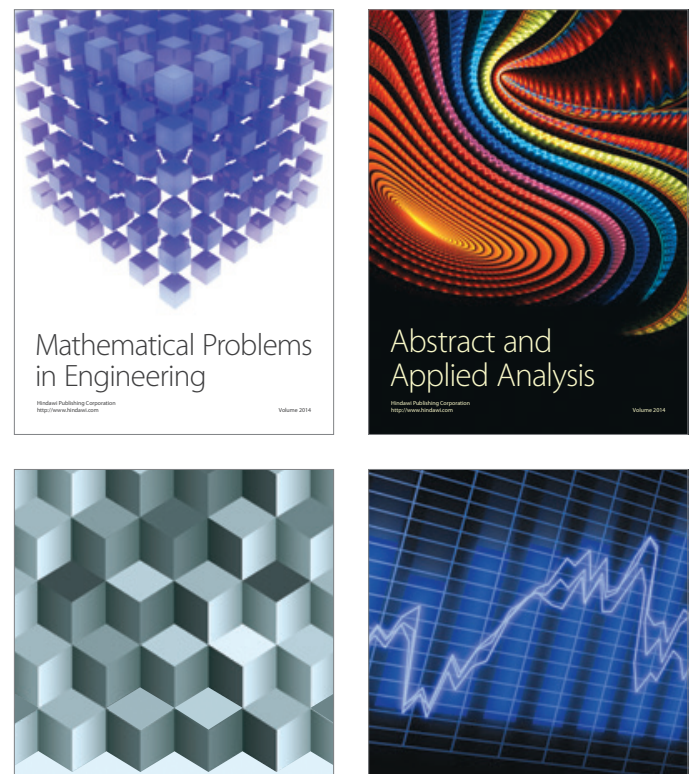

Journal of

Function Spaces

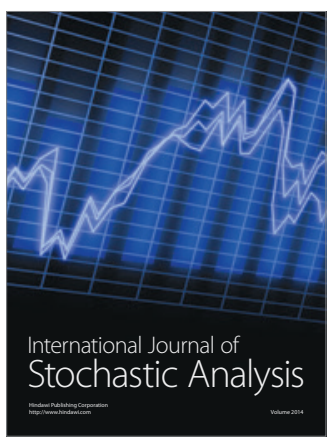

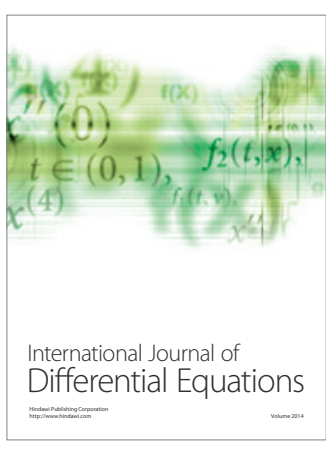
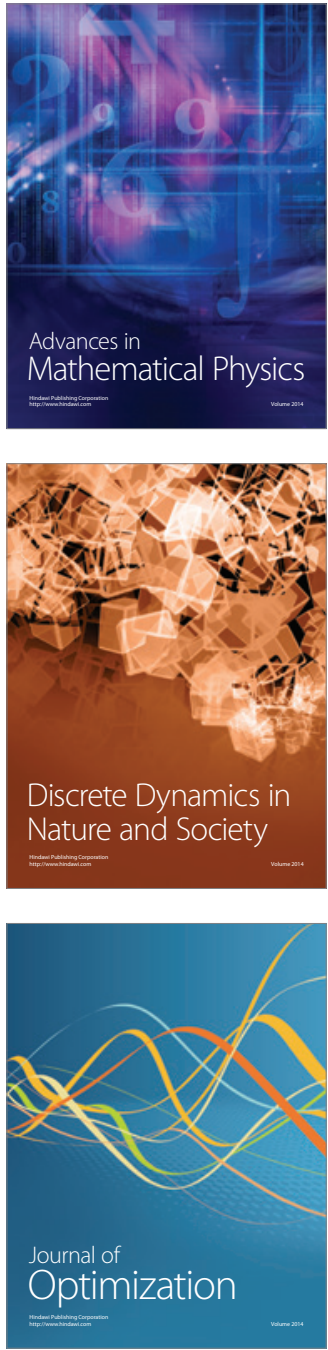